PROCEEDINGS OF THE AMERICAN MATHEMATICAL SOCIETY

Volume 128, Number 3, Pages 661-670

S 0002-9939(99)05224-7

Article electronically published on July 8, 1999

\title{
A MODULE-THEORETIC APPROACH TO CLIFFORD THEORY FOR BLOCKS
}

\author{
S. J. WITHERSPOON
}

(Communicated by Ronald M. Solomon)

\begin{abstract}
This work concerns a generalization of Clifford theory to blocks of group-graded algebras. A module-theoretic approach is taken to prove a one-to-one correspondence between the blocks of a fully group-graded algebra covering a given block of its identity component, and conjugacy classes of blocks of a twisted group algebra. In particular, this applies to blocks of a finite group covering blocks of a normal subgroup.
\end{abstract}

\section{INTRODUCTION}

In 1973 Dade developed the block theory of group-graded algebras, in particular extending the Clifford correspondence to blocks of group-graded algebras [9]. The Clifford correspondence for representations is a one-to-one correspondence between the irreducible representations of a group $G$ having a given constituent when restricted to a normal subgroup, and the irreducible projective representations (with respect to a particular 2-cocycle) of a certain subgroup of the corresponding quotient group [4]. Dade has recently used his results to make progress towards solving conjectures of Alperin and Dade $[12,13,15]$. Ellers has used Dade's work in making progress on a generalization of Alperin's Conjecture [16]. In view of this recent activity, it seems useful to provide an alternative approach to Dade's results. In this work we treat blocks as bimodules to provide a new proof of the Clifford correspondence for blocks of group-graded algebras. This approach clarifies the connection between the Clifford correspondence for blocks and that for representations.

Specifically, let $G$ be a finite group and $A$ a fully $G$-graded algebra of finite dimension over an algebraically closed field $k$. That is, $A=\sum_{g \in G} A_{g}$ is a direct sum of subspaces $A_{g}$ with $A_{g} A_{h}=A_{g h}$ for all $g, h \in G$. Here $A_{g} A_{h}$ denotes the set of all finite sums of products $x y$, where $x \in A_{g}$ and $y \in A_{h}$. The first example of a fully $G$-graded algebra is $A=k \Gamma$ for a group $\Gamma$ with normal subgroup $N$ and $G=\Gamma / N$. In this case the subspace $A_{g}$ is spanned by the elements of the coset $g$. As the identity component is $A_{1}=k N$, comparing blocks of $A_{1}$ to blocks of $A$ amounts to comparing blocks of $N$ to blocks of $\Gamma$.

We consider $A$ to be a right $A^{o p} \otimes A$-module, where $A^{o p}$ is the opposite algebra to $A$, via left and right multiplication by elements of $A$. A block of $A$ is an indecomposable direct summand of the $A^{o p} \otimes A$-module $A$. A block $\widetilde{B}$ of $A$ covers a block

Received by the editors April 20, 1998.

1991 Mathematics Subject Classification. Primary 20C20, 20C25.

Research supported in part by NSERC grant \# OGP0170281.

(C)1999 American Mathematical Society 
$B$ of $A_{1}$ if $B$ is a direct summand of the module $\widetilde{B}$ restricted to $A_{1}^{o p} \otimes A_{1}$. That this is equivalent to $\widetilde{B}$ lying over $B$ [9, p. 218] follows from the proof of Lemma 2.1 below. The group $G$ acts by conjugation on the blocks of $A_{1}$, an element $g \in G$ sending $B$ to $A_{g^{-1}} B A_{g}$. In Section 4 we assume $B$ is $G$-invariant and derive a one-to-one correspondence between the blocks of $A$ covering a given block $B$ of $A_{1}$ and $G$-conjugacy classes of blocks of a twisted group algebra for a subgroup of $G$ (Theorem 4.5 below). A reduction to this invariant case is given in Section 2.

A starting point for the Clifford correspondence for blocks is provided by a more general correspondence for indecomposable modules discussed in Section 3. In this context, we let $B \uparrow A_{1}^{o p} \otimes A$ denote the induced $A_{1}^{o p} \otimes A$-module $B \otimes A_{1}^{o p} \otimes A_{1}\left(A_{1}^{o p} \otimes A\right)$ and $\mathcal{E}=\operatorname{End}_{A_{1}^{o p} \otimes A}\left(B \uparrow^{A_{1}^{o p} \otimes A}\right)$. Then $\mathcal{E}$ is a (not necessarily fully) $G$-graded algebra; that is, $\mathcal{E}=\sum_{g \in G} \mathcal{E}_{g}$ is a direct sum of subspaces $\mathcal{E}_{g}$ with $\mathcal{E}_{g} \mathcal{E}_{h} \subseteq \mathcal{E}_{g h}$ for all $g, h \in G$. In Section 3 we describe the Miyashita action of $G$ on $\mathcal{E}$ which permutes the blocks. Let $J_{G}(\mathcal{E})$ denote the graded Jacobson radical of $\mathcal{E}$, the intersection of all maximal graded right ideals. We show in Section 4 that $\mathcal{E} / J_{G}(\mathcal{E})$ is a twisted group algebra for a subgroup of $G$, and prove the following theorem.

Theorem 4.5 (Clifford correspondence). Let $B$ be a $G$-invariant block of $A_{1}$. The blocks of $A$ covering $B$ correspond one-to-one with $G$-conjugacy classes of blocks of the twisted group algebra $\mathcal{E} / J_{G}(\mathcal{E})$.

In order to prove the theorem, in Section 3 we relate blocks of $A$ covering $B$ to blocks of the endomorphism algebra $\mathcal{E}$ in the following way. Let $\Delta$ be the diagonal subalgebra $\sum_{g \in G}\left(A_{g^{-1}}\right)^{o p} \otimes A_{g}$ of $A^{o p} \otimes A$. The identity component $A_{1}$ of $A$ is naturally a $\Delta$-module; we denote this $\Delta$-module by $\left(A_{1}\right)_{\Delta}$. The $A^{o p} \otimes A$ module $\left(A_{1}\right)_{\Delta} \uparrow A^{o p} \otimes A$ induced from $\Delta$ is isomorphic to $A$. If the block $B$ of $A_{1}$ is $G$-invariant, then $B$ is naturally a $\Delta$-module, and so we obtain an ideal direct summand $B_{\Delta} \uparrow A^{o p} \otimes A \cong A B A$ of $A$. We show in Section 2 that the blocks of $A$ covering $B$ are precisely the indecomposable direct summands of the $A^{o p} \otimes A$-module $B_{\Delta} \uparrow^{A^{o P} \otimes A}$. In turn, these direct summands are in one-to-one correspondence with the blocks of another endomorphism algebra, $\operatorname{End}_{A^{o p} \otimes A}\left(B_{\Delta} \uparrow A^{o p} \otimes A\right)$. This endomorphism algebra is isomorphic to the algebra $\mathcal{E}^{G}$ of fixed points of $\mathcal{E}=$ $\operatorname{End}_{A_{1}^{o p} \otimes A}\left(B \uparrow^{A_{1}^{o p} \otimes A}\right)$, and blocks of $\mathcal{E}^{G}$ correspond to $G$-conjugacy classes of blocks of $\mathcal{E}$, as we show in Section 3 .

Our results also hold in the more general situation where $k$ is replaced by a $p$-modular system $(K, R, k)$ for a prime $p$, where $k$ is algebraically closed. Here $R$ is a complete discrete valuation ring with maximal ideal $\mathfrak{p}$, quotient field $K$, and residue class field $k=R / \mathfrak{p}$ of characteristic $p$. The basic theory needed includes the Krull-Schmidt-Azumaya Theorem [7, Proposition 56.4] and existence of projective covers of modules which follows from [6, Theorem 6.23] and [7, Propositions 56.2 and 56.4]. In this situation, $A$ is a fully $G$-graded $R$-algebra (free and finitely generated as an $R$-module), and the twisted group algebra $\mathcal{E} / J_{G}(\mathcal{E})$ of the Clifford correspondence is a $k$-algebra as $J_{G}(\mathcal{E}) \supseteq \mathfrak{p} \mathcal{E}$. For simplicity however, we state our results in the case of a single base field $k$. All algebras and modules will be finite dimensional over $k$, and tensor products will be over $k$ unless otherwise indicated.

The author thanks J. L. Alperin for suggesting this work and P. R. Boisen, E. C. Dade, and H. Ellers for helpful conversations. 


\section{Reduction to the invariant CASE}

In this section we first give a correspondence between blocks covering a block $B$ of $A_{1}$ and blocks of the endomorphism algebra $\operatorname{End}_{A^{o p} \otimes A}\left(B_{\Delta_{B}} \uparrow^{o p} \otimes A\right)$. We then give a reduction to the invariant case. Let $G_{B}$ be the subgroup of $G$ fixing an arbitrary block $B$ of $A_{1}$, that is,

$$
G_{B}:=\left\{g \in G \mid A_{g^{-1}} B A_{g}=B\right\} .
$$

Let $A_{B}:=\sum_{g \in G_{B}} A_{g}$ and $\Delta_{B}:=\sum_{g \in G_{B}}\left(A^{o p}\right)_{g} \otimes A_{g}$, where $\left(A^{o p}\right)_{g}=\left(A_{g^{-1}}\right)^{o p}$. Then $\Delta_{B}$ is a fully $\delta\left(G_{B}\right)$-graded algebra where $\delta\left(G_{B}\right)=\left\{(g, g) \mid g \in G_{B}\right\} \subseteq G \times G$. Note that $B$ is naturally a $\Delta_{B}$-module, denoted $B_{\Delta_{B}}$. We observe that if $\widetilde{B}$ is a block of $A$ covering $B$, so that $B$ is a direct summand of $\widetilde{B} \downarrow_{A_{1}^{o p} \otimes A_{1}}$, then $\widetilde{B}$ covers all $G$-conjugates of $B$ : The restricted module $\widetilde{B} \downarrow_{A_{1}^{o p} \otimes A_{1}}$ is fixed by the $G$-action, as $\widetilde{B}$ is. So for any $g \in G, B^{g}=A_{g^{-1}} B A_{g}$ is also a direct summand of $\widetilde{B} \downarrow_{A_{1}^{o p} \otimes A_{1}}$. In addition $\widetilde{B}$ covers no other blocks of $A_{1}$, as we see in the following lemma. We refer the reader to [3], [10], or [18] for facts about conjugate modules and induced modules for a fully group-graded ring. The following lemma is analogous to $[1$, Lemma 4.3] or [7, Lemma $61.3(\mathrm{v})]$.

Lemma 2.1. Let $B$ be a block of $A_{1}$.

(i) The blocks of $A$ covering $B$ correspond one-to-one with the indecomposable summands of the $A^{o p} \otimes A$-module $B_{\Delta_{B}} \uparrow A^{o p} \otimes A$.

(ii) The blocks of $A$ covering $B$ correspond one-to-one with the blocks of the algebra $\operatorname{End}_{A^{o p} \otimes A}\left(B_{\triangle_{B} \uparrow A^{o p} \otimes A}\right)$.

(iii) If the block $\widetilde{B}$ of $A$ covers $B$, then $\widetilde{B}$ covers all $G$-conjugates of $B$ and no other blocks of $A_{1}$.

Proof. (i) Write $A_{1} \cong B_{1} \oplus \cdots \oplus B_{k} \oplus M$ as $A_{1}^{o p} \otimes A_{1}$-modules, where $B=B_{1}, \ldots, B_{k}$ are the distinct conjugates of $B$, and $M$ is the sum of the remaining blocks of $A_{1}$. The induced $A^{o p} \otimes A$-module $\left(A_{1}\right)_{\Delta} \uparrow A^{o p} \otimes A$ is isomorphic to $A$ [3, Lemma 3.3]; this isomorphism is given simply by sending an element $\sum_{i} a_{i} \otimes\left(b_{i} \otimes c_{i}\right)$ of $\left(A_{1}\right)_{\Delta} \uparrow A^{o p} \otimes A$ to $\sum_{i} b_{i} a_{i} c_{i}$. Similarly, $B_{\Delta_{B}} \uparrow^{A^{o p}} \otimes A \cong A B A$ as $A^{o p} \otimes A$-modules. As $B_{1} \oplus \cdots \oplus B_{k}$ and $M$ are naturally $\Delta$-submodules of $\left(A_{1}\right)_{\Delta}$, we have

$$
A \cong\left(B_{1} \oplus \cdots \oplus B_{k}\right)_{\Delta} \uparrow^{A^{o p} \otimes A} \oplus M_{\Delta} \uparrow^{A^{o p} \otimes A}
$$

as $A^{o p} \otimes A$-modules. The $\Delta$-module $\left(B_{1} \oplus \cdots \oplus B_{k}\right)_{\Delta}$ is isomorphic to $B_{\Delta_{B}} \uparrow^{\Delta}$ by an argument similar to that used in [3, Lemma 3.3]. Therefore $B_{\Delta_{B}} \uparrow A^{\Delta_{p} B} \otimes A \cong$ $B_{\Delta_{B}} \uparrow \Delta \uparrow A^{o p} \otimes A \cong\left(B_{1} \oplus \cdots \oplus B_{k}\right)_{\Delta} \uparrow A^{o p} \otimes A$, and so $A \cong B_{\Delta_{B}} \uparrow^{A^{o p} \otimes A} \oplus M_{\Delta} \uparrow A^{o p} \otimes A$.

Let $\widetilde{B}$ be a block of $A$ contained in $B_{\Delta_{B}} \uparrow A^{o p} \otimes A$. By Mackey's Theorem for group-graded rings [18, Theorem 8.4],

$$
B_{\Delta_{B}} \uparrow^{A^{o p} \otimes A_{\downarrow}}{ }_{A_{1}^{o p} \otimes A_{1}} \cong \sum_{(s, t) \in \delta\left(G_{B}\right) \backslash G \times G} B \otimes_{A_{1}^{o p} \otimes A_{1}}\left(\left(A^{o p}\right)_{s} \otimes A_{t}\right) .
$$

A similar argument to [3, Lemma 3.3] shows that the $A_{1}^{o p} \otimes A_{1}$-module $A_{s^{-1}} B A_{t}$ is isomorphic to the conjugate module $B \otimes A_{1}^{o p} \otimes A_{1}\left(\left(A^{o p}\right)_{s} \otimes A_{t}\right)$. As $B$ is indecomposable and $A$ is fully graded, these conjugate modules $A_{s^{-1}} B A_{t}$ are indecomposable as well. Since $\widetilde{B}$ divides $B_{\Delta_{B}} \uparrow^{A^{o p} \otimes A}$, the Krull-Schmidt Theorem now implies that some $A_{s^{-1}} B A_{t}$ divides $\widetilde{B} \downarrow_{A_{1}^{o p} \otimes A_{1}}$. Therefore $B$ is a summand of $A_{s}\left(\widetilde{B} \downarrow_{A_{1}^{o p} \otimes A_{1}}\right) A_{t^{-1}} \cong \widetilde{B} \downarrow_{A_{1}^{o p} \otimes A_{1}}$; that is, $\widetilde{B}$ covers $B$. 
Now assume $\widetilde{B}$ is a block of $A$ covering $B$, but does not divide $B_{\Delta_{B}} \uparrow^{A^{o p} \otimes A}$. Then $\widetilde{B}$ divides $M_{\Delta} \uparrow A^{o p} \otimes A$, and so $B$ divides $M_{\Delta} \uparrow A^{o p} \otimes A_{\downarrow_{1}^{o p} \otimes A_{1}}$. As before, we apply Mackey's Theorem to $M_{\Delta} \uparrow^{A^{o p} \otimes A} \downarrow_{A_{1}^{o p} \otimes A_{1}}$, and conclude that $B$ divides $A_{s^{-1}} M A_{t}$ for some $s, t \in G$. This implies that the conjugate $A_{1}^{o p} \otimes A_{1}$-module $B^{s^{-1}}=$ $A_{s} B A_{s^{-1}}$ divides $A_{s} A_{s^{-1}} M A_{t} A_{s^{-1}}=M A_{t s^{-1}}$. Letting $e$ be the primitive central idempotent of $A_{1}$ corresponding to the block $B^{s^{-1}}$, we derive a contradiction, as $e B^{s^{-1}} \neq 0$ and $e M=0$. Therefore the blocks of $A$ covering $B$ are exactly the indecomposable summands of $B_{\Delta_{B}} \uparrow A^{o p} \otimes A$.

(ii) As a summand of $A, B_{\Delta_{B}} \uparrow A^{o p} \otimes A$ is an algebra, and

$$
\operatorname{End}_{A^{o p} \otimes A}\left(B_{\Delta_{B}} \uparrow^{o p} \otimes A\right) \cong Z\left(B_{\Delta_{B}} \uparrow^{o p} \otimes A\right),
$$

the center of $B_{\Delta_{B}} \uparrow A^{o p} \otimes A$. Thus blocks of $B_{\Delta_{B}} \uparrow A^{o p} \otimes A$ correspond one-to-one with blocks of $\operatorname{End}_{A^{o p} \otimes A}\left(B_{\Delta_{B}} \uparrow^{o p} \otimes A\right)$.

(iii) This follows immediately from the proof of (i) and the observation preceding the lemma.

We next show that the blocks of $A$ covering $B$ correspond one-to-one with the blocks of $A_{B}$ covering $B$, a reduction allowing us to consider only the $G$-invariant case from now on. This is a version of the Fong-Reynolds Theorem [17, V.2.5].

Lemma 2.2. Let $B$ be a block of $A_{1}$. The blocks of $A$ covering $B$ correspond oneto-one with the blocks of $A_{B}$ covering $B$. Further, this correspondence is given by induction of blocks of $A_{B}$, as $A_{B}^{o p} \otimes A_{B}$-modules, to $A^{o p} \otimes A$-modules.

Proof. We prove the first statement by showing that there is an isomorphism of algebras

$$
\operatorname{End}_{A_{B}^{o p} \otimes A_{B}}\left(B_{\Delta_{B}} \uparrow^{A_{B}^{o p} \otimes A_{B}}\right) \cong \operatorname{End}_{A^{o p} \otimes A}\left(B_{\Delta_{B}} \uparrow^{A^{o p} \otimes A}\right),
$$

and applying Lemma 2.1 (ii). By the Nakayama relations [2, Proposition 2.8.3] we have two natural isomorphisms

$$
\operatorname{End}_{A_{B}^{o p} \otimes A_{B}}\left(B_{\Delta_{B}} \uparrow A_{B}^{o p} \otimes A_{B}\right) \cong \operatorname{Hom}_{\Delta_{B}}\left(B_{\Delta_{B}}, B_{\Delta_{B}} \uparrow A_{B}^{o p} \otimes A_{B} \downarrow_{\Delta_{B}}\right)
$$

and

$$
\operatorname{End}_{A^{o p} \otimes A}\left(B_{\Delta_{B}} \uparrow^{A^{o p} \otimes A}\right) \cong \operatorname{Hom}_{\Delta_{B}}\left(B_{\Delta_{B}}, B_{\Delta_{B}} \uparrow^{A^{o p} \otimes A_{\downarrow_{\Delta_{B}}}}\right) .
$$

We may consider $B_{\Delta_{B}} \uparrow A_{B}^{o p} \otimes A_{B} \downarrow_{\Delta_{B}}$ as a direct summand of $B_{\Delta_{B}} \uparrow A^{o p} \otimes A \downarrow_{\Delta_{B}}$ by Mackey's Theorem [18, Theorem 8.4]. In order to achieve the desired isomorphism, we need only show that all $\Delta_{B}$-homomorphisms from $B_{\Delta_{B}}$ to $B_{\Delta_{B}} \uparrow A^{o p} \otimes A_{\downarrow_{\Delta_{B}}}$ in fact have image contained in $B_{\Delta_{B}} \uparrow A_{B}^{o p} \otimes A_{B} \downarrow_{\Delta_{B}}$. Consider a summand $A_{s} B A_{t}=$ $A_{s} B A_{s^{-1}} A_{s t}=B^{s^{-1}} A_{s t}$ of $B_{\Delta_{B}} \uparrow^{A^{o p} \otimes A_{\downarrow_{\Delta_{B}}}}$ as an $A_{1}^{o p} \otimes A_{1}$-module. If $s \notin G_{B}$, then the primitive central idempotent $e$ of $A_{1}$ associated with $B$ yields the identity map on $B$ but 0 on $B^{s^{-1}} \neq B$. In this case there are no $A_{1}^{o p} \otimes A_{1}$-homomorphisms, and so no $\Delta_{B}$-homomorphisms from $B_{\Delta_{B}}$ to $A_{s} B A_{t}$. A similar argument works if $s \in G_{B}$ and $t \notin G_{B}$. Therefore we have proved the first statement of the lemma.

By Lemma 2.1 (i), blocks of $A_{B}$ covering $B$ are the indecomposable $A_{B}^{o p} \otimes A_{B^{-}}$ direct summands of $B_{\Delta_{B}} \uparrow_{B}^{o p} \otimes A_{B}$, while blocks of $A$ covering $B$ are the indecomposable $A^{\text {op }} \otimes A$-direct summands of

$$
B_{\Delta_{B}} \uparrow^{A^{o p}} \otimes A \cong\left(B_{\Delta_{B}} \uparrow^{A_{B}^{o p} \otimes A_{B}}\right) \uparrow A^{o p} \otimes A .
$$


Suppose that $B_{\Delta_{B}} \uparrow A_{B}^{o p} \otimes A_{B} \cong \widetilde{B}_{1} \oplus \cdots \oplus \widetilde{B}_{n}$ is a decomposition into indecomposable $A_{B}^{o p} \otimes A_{B}$-modules. Then

$$
B_{\Delta_{B}} \uparrow^{A^{o p} \otimes A} \cong \widetilde{B}_{1} \uparrow^{A^{o p} \otimes A} \oplus \cdots \oplus \widetilde{B}_{n} \uparrow^{A^{o p} \otimes A}
$$

as $A^{o p} \otimes A$-modules. If some $\widetilde{B}_{i} \uparrow^{A^{o p} \otimes A}$ were not indecomposable, there would be a contradiction to the first statement of the lemma. Therefore each $\widetilde{B}_{i} \uparrow A^{o p} \otimes A$ is a block of $A$.

\section{TWO ENDOMORPHISM ALGEBRAS}

In this section we assume $B$ is a $G$-invariant block of $A_{1}$, so that $G_{B}=G$, $A_{B}=A$, and $\Delta_{B}=\Delta=\sum_{g \in G}\left(A^{o p}\right)_{g} \otimes A_{g}$.

We first discuss a Clifford correspondence for indecomposable modules which is implicit in Dade's work [14]. This provides a starting point for the Clifford correspondence for blocks. We show that an endomorphism algebra arising in this indecomposable module situation (as applied to a block) is in fact the fixed point subalgebra of the endomorphism algebra of Lemma 2.1 (ii) under an appropriate $G$-action. It seems necessary to look at both endomorphism algebras to achieve the Clifford correspondence for blocks from this point of view. Moreover this development clarifies the connection to the classical Clifford correspondence, and explains why $G$-conjugacy classes arise in the Clifford correspondence for blocks.

Let $V$ be an indecomposable right $A_{1}$-module, and $\mathcal{E}=\operatorname{End}_{A}\left(V \uparrow^{A}\right)$, where $V \uparrow^{A}=V \otimes_{A_{1}} A$ is the induced $A$-module. As $V \uparrow^{A}$ is a graded module [20], $\mathcal{E}$ is a $G$-graded algebra with identity component $\mathcal{E}_{1} \cong \operatorname{End}_{A_{1}}(V)$. One way to see this is to consider the isomorphisms of vector spaces

$$
\mathcal{E} \cong \operatorname{Hom}_{A_{1}}\left(V, V \uparrow^{A_{\downarrow}}{ }_{A_{1}}\right) \cong \sum_{g \in G} \operatorname{Hom}_{A_{1}}\left(V, V^{g}\right)
$$

that follow from [2, Proposition 2.8.3] and Mackey's Theorem [18, Theorem 8.4]. Here $V^{g}$ is the $A_{1}$-module $V \otimes_{A_{1}} A_{g}$, and $\mathcal{E}_{g} \cong \operatorname{Hom}_{A_{1}}\left(V, V^{g}\right)$ as a vector space. If $V$ is $G$-invariant, so that $V^{g} \cong V$ for all $g \in G$, then $\mathcal{E}$ is fully $G$-graded, as may be seen from the above decomposition of $\mathcal{E}$ or [20, I.5.2]. If $V$ is also irreducible, then Schur's Lemma implies that $\mathcal{E}$ is a twisted group algebra. This is the twisted group algebra of the classical Clifford correspondence. In case $V$ is indecomposable but not irreducible, or in case $k$ is replaced by a complete discrete valuation ring $R$, the fact that $\mathcal{E}_{1} \cong \operatorname{End}_{A_{1}}(V)$ is local [6, Proposition 6.10] replaces Schur's Lemma as follows: Let $J_{G}(\mathcal{E})$ denote the graded Jacobson radical of $\mathcal{E}$, the intersection of all maximal graded right ideals. By $\left[5\right.$, Theorem 4.4] $J_{G}(\mathcal{E})$ is contained in the ordinary Jacobson radical $J(\mathcal{E})$. In case $V$ is $G$-invariant, [14, Proposition 2.19], [6, Proposition 5.22], and [8, Lemma 14.2] imply that $\mathcal{E} / J_{G}(\mathcal{E})$ is a twisted group algebra for a subgroup of $G$. For the sake of completeness, we will give these arguments in greater detail later for the special case where $V$ is a block.

Whether or not $V$ is $G$-invariant, there is a one-to-one correspondence between indecomposable $A$-direct summands of $V \uparrow^{A}$ and indecomposable right $\mathcal{E} / J_{G}(\mathcal{E})$ direct summands of $\mathcal{E} / J_{G}(\mathcal{E})$ : Indecomposable summands of $V \uparrow^{A}$ correspond oneto-one with indecomposable right summands of $\mathcal{E}$ by sending a summand of $V \uparrow^{A}$ to the corresponding projection endomorphism, an idempotent of $\mathcal{E}$ [6, Proposition 6.3]. As $J_{G}(\mathcal{E}) \subseteq J(\mathcal{E})$, indecomposable right $\mathcal{E}$-direct summands of $\mathcal{E}$ correspond with indecomposable right $\mathcal{E} / J_{G}(\mathcal{E})$-direct summands of $\mathcal{E} / J_{G}(\mathcal{E})[6$, Corollaries 6.22 and 6.25$]$. 
In the block situation, we replace $A$ by the fully $G$-graded algebra $A_{1}^{o p} \otimes A, A_{1}$ by $A_{1}^{o p} \otimes A_{1}$, and $V$ by the block $B$ of $A_{1}$. (We remark that replacing $A$ by $A^{o p} \otimes A_{1}$ would work equally well.) The relevant $G$-graded endomorphism algebra is then

$$
\mathcal{E}:=\operatorname{End}_{A_{1}^{o p} \otimes A}\left(B \uparrow A_{1}^{o p} \otimes A\right)
$$

and the Clifford correspondence for indecomposable modules described above applies here. It will need to be modified to yield the Clifford correspondence for blocks given in Theorem 4.5. We caution that, although $B$ is $G$-invariant as a block, it may not be $1 \times G$-invariant as an $A_{1}^{o p} \otimes A_{1}$-module. Thus it does not follow from the above arguments that $\mathcal{E} / J_{G}(\mathcal{E})$ is a twisted group algebra; in Section 4 we show that this fact follows from a characterization of the graded Jacobson radical given in [14]. In addition, we are interested in $A^{o p} \otimes A$-modules rather than $A_{1}^{o p} \otimes A$-modules. This is where a $G$-action on $\mathcal{E}$ arises. The following proposition is Theorem 1.3 of [19]; see also [11, Theorem 2.1].

Proposition 3.1 (Miyashita action). Let $G$ be a finite group and $S$ a fully $G$ graded algebra. Let $M$ and $N$ be $S$-modules, $g \in G$, and $\phi \in \operatorname{Hom}_{S_{1}}(M, N)$. Then there is a unique element $\phi^{g} \in \operatorname{Hom}_{S_{1}}(M, N)$ such that $\phi^{g}\left(m s_{g}\right)=\phi(m) s_{g}$ for all $m \in M$ and $s_{g} \in S_{g}$. If $M=N$, then $G$ acts as algebra automorphisms of $\operatorname{End}_{S_{1}}(M)$.

We give the definition of $\phi^{g}$, which will be needed in the next section. As $S$ is fully $G$-graded, we have $S_{g^{-1}} S_{g}=S_{1}$ for all $g \in G$. As $1 \in S_{1}$ [20], there are elements $\alpha_{i} \in S_{g^{-1}}, \beta_{i} \in S_{g}$ such that $\sum_{i=1}^{n} \alpha_{i} \beta_{i}=1$. Then, for all $m \in M$,

$$
\phi^{g}(m):=\sum_{i=1}^{n} \phi\left(m \alpha_{i}\right) \beta_{i} .
$$

Now let $S=A^{o p} \otimes A$ be the fully $G$-graded algebra with $S_{g}=\left(A^{o p}\right)_{g} \otimes A$. The proposition gives a $G$-action on $\operatorname{End}_{A_{1}^{o p} \otimes A}\left(B_{\Delta} \uparrow^{A^{o p} \otimes A}\right)$, where $\Delta=\sum_{g \in G}\left(A^{o p}\right)_{g} \otimes$ $A_{g}$, and $B_{\Delta}$ is the $\Delta$-module $B$. This provides the connection between $\mathcal{E}=$ End $_{A_{1}^{o p} \otimes A}\left(B \uparrow^{o p} \otimes A\right)$ and the algebra $\operatorname{End}_{A^{o p} \otimes A}\left(B_{\Delta} \uparrow^{A^{o p} \otimes A}\right)$ of Lemma 2.1 (ii), as we see next. We note that $B_{\Delta} \uparrow^{A^{o p} \otimes A} \downarrow_{A_{1}^{o p} \otimes A} \cong B \uparrow^{A_{1}^{o p} \otimes A}$, where the latter module is induced from $A_{1}^{o p} \otimes A_{1}$. This follows from an application of Mackey's Theorem [18, Theorem 8.4], as there is only one $\delta(G), 1 \times G$-double coset in $G \times G$, and $B_{\Delta} \downarrow_{A_{1}^{o p} \otimes A_{1}}=B$. Therefore

$$
\operatorname{End}_{A_{1}^{o p} \otimes A}\left(B_{\Delta} \uparrow A^{o p} \otimes A\right) \cong \operatorname{End}_{A_{1}^{o p} \otimes A}\left(B \uparrow^{A_{1}^{o p} \otimes A}\right)=\mathcal{E}
$$

Thus the above proposition yields an action of $G$ as automorphisms of $\mathcal{E}$. The next lemma describing the fixed point subalgebra $\mathcal{E}^{G}$ follows immediately.

Lemma 3.2. $\mathcal{E}^{G} \cong \operatorname{End}_{A^{o p} \otimes A}\left(B_{\Delta} \uparrow A^{o p} \otimes A\right)$.

Note that the second endomorphism algebra in the lemma is the algebra of Lemma 2.1 (ii). We mention that if we had taken $S_{g}=A^{o p} \otimes A_{g}$ instead, we would have gotten a different $G$-graded endomorphism algebra, but upon taking $G$-fixed points, we would have obtained the same $A^{o p} \otimes A$-endomorphism algebra.

We now give the relationship between our endomorphism algebras and the centralizer algebras of [9]. Let $C_{A}\left(A_{1}\right)$ denote the centralizer in $A$ of $A_{1}$. Let $e$ be the primitive central idempotent of $A_{1}$ corresponding to the block $B$. Then as graded algebras, $\mathcal{E}$ is isomorphic to $e C_{A}\left(A_{1}\right)$ : We identify $B \uparrow^{A_{1}^{o p} \otimes A}$ with 
$\sum_{g \in G} B A_{g}$ as in the proof of Lemma 2.1 (i). Given $\phi \in \mathcal{E}, \phi$ is determined by $\phi(e)$, as $\phi(b)=\phi(e b)=\phi(e) b$ for any $b \in B \uparrow A_{1}^{o p} \otimes A$. Further, $\phi(e) \in e C_{A}\left(A_{1}\right)$ as $e \phi(e)=\phi\left(e^{2}\right)=\phi(e)$ and $\phi \in \mathcal{E}$ implies that $\phi(e)$ commutes with elements of $A_{1}$. Conversely, any element of $e C_{A}\left(A_{1}\right)$ defines an element of $\mathcal{E}$ in this way. The $G$-grading on $e C_{A}\left(A_{1}\right)$ inherited from $A$ corresponds to that of $\mathcal{E}$, and the $G$-action on $\mathcal{E}$ provided by Proposition 3.1 gives rise to a $G$-action on $e C_{A}\left(A_{1}\right)$, which is just the usual Miyashita action on this algebra.

Lemma 3.3. The subalgebras $\mathcal{E}^{G}$ and $\mathcal{E}_{1}$ of $\mathcal{E}$ are both contained in the center of $\mathcal{E}$.

Proof. Let $\phi \in \mathcal{E}$ and $\psi \in \mathcal{E}_{g}$ for some $g \in G$, and suppose $\psi(e)=r_{g} \in e C_{A}\left(A_{1}\right)$, so that $\psi(b)=r_{g} b$ for all $b \in B \uparrow A_{1}^{o p} \otimes A$. Applying Proposition 3.1, $\phi^{g^{-1}} \circ \psi(e)=$ $\phi^{g^{-1}}\left(r_{g} e\right)=r_{g} \phi(e)=\psi \circ \phi(e)$, so that $\phi^{g^{-1}} \circ \psi=\psi \circ \phi$. If $\phi \in \mathcal{E}^{G}$, then $\phi$ is in the center of $\mathcal{E}$. If $\psi \in \mathcal{E}_{1}$, then $\phi \circ \psi=\psi \circ \phi$ for all $\phi \in \mathcal{E}$, so that $\psi$ is in the center of $\mathcal{E}$.

The next lemma shows that blocks of $\mathcal{E}^{G}$ correspond one-to-one to $G$-conjugacy classes of blocks of $\mathcal{E}$, as $\mathcal{E}^{G}$ is central in $\mathcal{E}$ by the previous lemma. Combined with Lemma 2.1 (ii) for $G=G_{B}$, this shows that blocks of $A$ covering $B$ correspond one-to-one with $G$-conjugacy classes of blocks of $\mathcal{E}$.

Lemma 3.4. Let $S$ be a finite dimensional $k$-algebra with an action of $G$ as automorphisms, and suppose that $S^{G}$ is central in $S$. Then the blocks of $S^{G}$ correspond one-to-one with the $G$-conjugacy classes of blocks of $S$.

Proof. A $G$-conjugacy class of blocks of $S$ corresponds to a $G$-conjugacy class $e_{1}, \ldots, e_{k}$ of primitive central idempotents of $S$. Their sum $e^{\prime}=e_{1}+\cdots e_{k}$ is a central idempotent of $S^{G}$. If $e^{\prime}$ were not primitive in $S^{G}$, then it would decompose into a sum of primitive central idempotents in $S^{G}$, each of which is a central idempotent of $S$ as $S^{G}$ is central in $S$. Thus each decomposes into a sum of primitive central idempotents of $S$, and by uniqueness these must be the $e_{1}, \ldots, e_{k}$. This contradicts the assumption that $e_{1}, \ldots, e_{k}$ is a single $G$-conjugacy class. Conversely, a block of $S^{G}$ corresponds to a primitive central idempotent of $S^{G}$, which is a central idempotent of $S$, and decomposes into primitive central idempotents of $S$. The $G$-action must permute these primitive central idempotents transitively.

\section{The Clifford correspondence}

We continue under the assumption that $B$ is a $G$-invariant block of $A_{1}$. In this section we show that blocks of $\mathcal{E}=\operatorname{End}_{A_{1}^{o p} \otimes A}\left(B \uparrow A_{1}^{o p} \otimes A\right)$ correspond one-to-one with blocks of $\mathcal{E} / J_{G}(\mathcal{E})$, a version of [9, Lemma 3.1 and Theorem 3.5]. Together with the results of Sections 2 and 3, this yields the Clifford correspondence in Theorem 4.5. In order to achieve this correspondence, it seems necessary to introduce a fully group-graded subalgebra $\mathcal{E}[B]$ of $\mathcal{E}$, as is done for centralizer algebras in [9].

Let $G[B]$ be the subgroup of $G$ defined by $g \in G[B]$ if and only if $\mathcal{E}_{g}$ contains a unit, and

$$
\mathcal{E}[B]:=\mathcal{E}_{G[B]}=\sum_{g \in G[B]} \mathcal{E}_{g} .
$$

Then $\mathcal{E}[B]$ is a fully $G[B]$-graded algebra, as for each $g \in G[B], \mathcal{E}_{g}=u_{g} \mathcal{E}_{1}$ for a unit $u_{g} \in \mathcal{E}_{g}$. There are examples for which $G[B] \neq G$ [16, Lemma 3.11]. 
The graded Jacobson radical $J_{G}(\mathcal{E})$ of any group-graded algebra $\mathcal{E}$ is a graded two-sided ideal of $\mathcal{E}$ [20, Lemma I.7.4]. We give the characterization of $J_{G}(\mathcal{E})$ in [14], providing details for the sake of completeness. The components of $J_{G}(\mathcal{E})$ are given by

$$
J_{G}(\mathcal{E})_{g}=\left\{\phi \in \mathcal{E}_{g} \mid \phi \mathcal{E}_{g^{-1}} \subseteq J\left(\mathcal{E}_{1}\right)\right\} .
$$

This follows immediately from the one-to-one correspondence between all maximal $G$-graded right ideals $M$ of $\mathcal{E}$ and all maximal right ideals $N$ of $\mathcal{E}_{1}$ given by sending $M$ to $N=M_{1}$, and $N$ to $M$ where $M_{g}=\left\{\phi \in \mathcal{E}_{g} \mid \phi \mathcal{E}_{g^{-1}} \subseteq N\right\}$. This correspondence is straightforward to verify. $A s B$ is an indecomposable $A_{1}^{o p} \otimes A_{1}$-module, $\mathcal{E}_{1} \cong \operatorname{End}_{A_{1}^{o p} \otimes A_{1}}(B)$ is local, and so $\mathcal{E}_{g}-J_{G}(\mathcal{E})_{g}$ is the set of units in $\mathcal{E}_{g}[14$, Lemma 2.18]. It follows that

$$
J_{G}(\mathcal{E})_{g}= \begin{cases}J\left(\mathcal{E}_{1}\right) \mathcal{E}_{g} & \text { if } g \in G[B], \\ \mathcal{E}_{g} & \text { if } g \notin G[B],\end{cases}
$$

as in [14, Proposition 2.19]. These arguments also apply to $\mathcal{E}[B]$, so in that case $J_{G[B]}(\mathcal{E}[B])=J\left(\mathcal{E}_{1}\right) \mathcal{E}[B]$, and $\mathcal{E}[B] / J_{G[B]}(\mathcal{E}[B])$ is a twisted group algebra for $G[B]$ by $[8$, Lemma 14.2$]$. The above characterizations of $J_{G}(\mathcal{E})$ and $J_{G[B]}(\mathcal{E}[B])$ also immediately imply the following lemma.

Lemma 4.1. There is an isomorphism of twisted group algebras

$$
\mathcal{E} / J_{G}(\mathcal{E}) \cong \mathcal{E}[B] / J_{G[B]}(\mathcal{E}[B]) .
$$

The next lemma, a generalization of [9, Lemma 3.1], may be applied to our situation with $H=G[B]$ and $S=\mathcal{E}[B]$ as $\mathcal{E}_{1}$ is central in $\mathcal{E}[B]$ by Lemma 3.3, and $J_{G[B]}(\mathcal{E}[B])=J\left(\mathcal{E}_{1}\right) \mathcal{E}[B]$. This shows the necessity of dealing with $\mathcal{E}[B]$ rather than $\mathcal{E}$.

Lemma 4.2. Let $H$ be a finite group, and $S$ a fully $H$-graded algebra in which $S_{1}$ is central and $J_{H}(S)=J\left(S_{1}\right) S$. Then the blocks of $S$ correspond one-to-one with blocks of $S / J_{H}(S)$.

Proof. Let $S / J_{H}(S)=b_{1} \oplus \cdots \oplus b_{k}$ be a decomposition into blocks. Consider each $b_{i}$ as a right $S$-module via the quotient map from $S$ to $S / J_{H}(S)$. Let $P\left(b_{i}\right)$ be the projective cover of $b_{i}$ as a right $S$-module. As $J_{H}(S) \subseteq J(S)$ [5, Theorem 4.4], $S$ itself is the projective cover of $S / J_{H}(S)$ as a right $S$-module [6, Corollary 6.22]. As projective covers preserve direct sums, we have $S \cong P\left(b_{1}\right) \oplus \cdots P\left(b_{k}\right)$ as a right $S$-module. We identify the $P\left(b_{i}\right)$ with right ideals of $S$ via this isomorphism. We will show that the $P\left(b_{i}\right)$ are also left ideals, and that they are indecomposable $S^{o p} \otimes S$-modules; that is, the $P\left(b_{i}\right)$ are blocks of $S$.

We let $P^{\prime}\left(b_{i}\right)$ be the projective cover of $b_{i}$ as a left $S$-module, so that $S \cong$ $P^{\prime}\left(b_{1}\right) \oplus \cdots \oplus P^{\prime}\left(b_{k}\right)$ as a left $S$-module. Consider the sum $m_{i}$ of all blocks $b_{j}$ with $j \neq i$. We have $S \cong P\left(b_{i}\right) \oplus P\left(m_{i}\right)$ as a right $S$-module, and $S \cong P^{\prime}\left(b_{i}\right) \oplus P^{\prime}\left(m_{i}\right)$ as a left $S$-module. Restrict these $S$-modules to $S_{1}$-modules. As $S_{1}$ is central in $S$, we do not distinguish between left and right $S_{1}$-modules. We have $P\left(b_{i}\right)=$ $P\left(b_{i}\right) S \cong P\left(b_{i}\right) P^{\prime}\left(b_{i}\right) \oplus P\left(b_{i}\right) P^{\prime}\left(m_{i}\right)$ and $P\left(m_{i}\right) \cong P\left(m_{i}\right) P^{\prime}\left(b_{i}\right) \oplus P\left(m_{i}\right) P^{\prime}\left(m_{i}\right)$ as $S_{1}$-modules. Therefore, as $S_{1}$-modules,

$$
S \cong P\left(b_{i}\right) P^{\prime}\left(b_{i}\right) \oplus P\left(b_{i}\right) P^{\prime}\left(m_{i}\right) \oplus P\left(m_{i}\right) P^{\prime}\left(b_{i}\right) \oplus P\left(m_{i}\right) P^{\prime}\left(m_{i}\right) .
$$

The right $S_{1}$-module $P\left(b_{i}\right) P^{\prime}\left(m_{i}\right)$ becomes 0 on passing to the quotient $S / J_{H}(S)$. As $J_{H}(S)=J\left(S_{1}\right) S$, it follows that $J\left(S_{1}\right) P\left(b_{i}\right) P^{\prime}\left(m_{i}\right)=P\left(b_{i}\right) P^{\prime}\left(m_{i}\right)$. Therefore 
by Nakayama's Lemma, $P\left(b_{i}\right) P^{\prime}\left(m_{i}\right)=0$, and similarly $P\left(m_{i}\right) P^{\prime}\left(b_{i}\right)=0$. Thus

$$
S \cong P\left(b_{i}\right) P^{\prime}\left(b_{i}\right) \oplus P\left(m_{i}\right) P^{\prime}\left(m_{i}\right)
$$

as $S_{1}$-modules, where $P\left(b_{i}\right)=P\left(b_{i}\right) P^{\prime}\left(b_{i}\right)=P^{\prime}\left(b_{i}\right)$, and $P\left(m_{i}\right)=P\left(m_{i}\right) P^{\prime}\left(m_{i}\right)=$ $P^{\prime}\left(m_{i}\right)$. It follows that each $P\left(b_{i}\right)$ is both a left and a right ideal of $S$.

Suppose $P\left(b_{i}\right)=B_{1} \oplus B_{2}$ as $S^{o p} \otimes S$-modules. Under the quotient map from $S$ to $S / J_{H}(S)$, the image of one of $B_{1}, B_{2}$ must be 0 . Considering $B_{1}$ and $B_{2}$ as $S_{1}$-modules, another application of Nakayama's Lemma implies that one of $B_{1}, B_{2}$ is 0 .

Next we see that $G$-conjugacy classes of blocks of $\mathcal{E} / J_{G}(\mathcal{E})$ correspond one-to-one with $G$-conjugacy classes of blocks of $\mathcal{E}$, resulting in the Clifford correspondence. For this, we need a lemma.

Lemma 4.3. The subgroup $G[B]$ is normal in $G$, and $\left(\mathcal{E}_{h}\right)^{g}=\mathcal{E}_{h^{g}}$ for all $g, h \in G$.

Proof. Let $g, h \in G$, and $\phi \in \mathcal{E}_{h}$. By (3.1), $\phi^{g}(b)=\sum_{i} \beta_{i} \phi\left(\alpha_{i} b\right)$ for all $b \in$ $B \uparrow A_{1}^{o p} \otimes A$, where $\alpha_{i} \in A_{g}, \beta_{i} \in A_{g^{-1}}$, and $\sum_{i} \beta_{i} \alpha_{i}=1$. Let $e$ be the primitive central idempotent of $A_{1}$ corresponding to the block $B$, so $\phi(e) \in A_{h}$ as discussed in the text preceding Lemma 3.3. As $e$ is a $G$-invariant element of $C_{A}\left(A_{1}\right), e$ is central in $A_{1}$ so $\phi^{g}(e)=\sum_{i} \beta_{i} \phi\left(\alpha_{i} e\right)=\sum_{i} \beta_{i} \phi(e) \alpha_{i}$, since $\phi$ is an $A_{1}^{o p} \otimes A$-map. But $\sum_{i} \beta_{i} \phi(e) \alpha_{i} \in A_{g^{-1}} A_{h} A_{g}=A_{h^{g}}$. Therefore $\phi^{g} \in \mathcal{E}_{h^{g}}$, and $\left(\mathcal{E}_{h}\right)^{g} \subseteq \mathcal{E}_{h^{g}}$. Conversely, let $\psi \in \mathcal{E}_{h^{g}}$, and let $\phi=\psi^{g^{-1}} \in\left(\mathcal{E}_{h^{g}}\right)^{g^{-1}} \subseteq \mathcal{E}_{h}$ by the above argument. Then $\psi=\phi^{g} \in\left(\mathcal{E}_{h}\right)^{g}$, and so $\mathcal{E}_{h^{g}}=\left(\mathcal{E}_{h}\right)^{g}$.

To see that $G[B]$ is normal in $G$, let $h \in G[B], g \in G$, and $u_{h}$ a unit in $\mathcal{E}_{h}$. Then $\left(u_{h}\right)^{g}$ is a unit in $\mathcal{E}_{h^{g}}$.

By the lemma, the $G$-action on $\mathcal{E}$ yields a $G$-action on $\mathcal{E}[B]$. Further, $G$ fixes the ideal $J_{G[B]}(\mathcal{E}[B])=J\left(\mathcal{E}_{1}\right) \mathcal{E}[B]$, as $G$ fixes $\mathcal{E}_{1}$ by the lemma and so permutes the maximal ideals of $\mathcal{E}_{1}$. Therefore the $G$-action on $\mathcal{E}[B]$ induces a $G$-action on $\mathcal{E}[B] / J_{G[B]}(\mathcal{E}[B])$.

Lemma 4.4. The $G$-conjugacy classes of blocks of $\mathcal{E}$ correspond one-to-one with $G$-conjugacy classes of blocks of $\mathcal{E} / J_{G}(\mathcal{E})$.

Proof. By Lemmas 4.1 and 4.2, we need only show that $G$-conjugacy classes of blocks of $\mathcal{E}[B]$ correspond one-to-one with $G$-conjugacy classes of blocks of $\mathcal{E}$. Given a $G$-conjugacy class of blocks of $\mathcal{E}[B]$, there is a corresponding $G$-invariant central idempotent $e$ of $\mathcal{E}[B]$, which is also then a $G$-invariant central idempotent of $\mathcal{E}$, as $\mathcal{E}^{G}$ is central in $\mathcal{E}$. Consider the image $\bar{e}$ of $e$ in $\mathcal{E} / J_{G}(\mathcal{E}) \cong \mathcal{E}[B] / J_{G[B]}(\mathcal{E}[B])$. If $e$ may be decomposed in $\mathcal{E}$ as the sum of two nontrivial $G$-invariant central idempotents, this decomposition gives such a decomposition of $\bar{e}$, contradicting Lemma 4.2.

Conversely, a $G$-conjugacy class of blocks of $\mathcal{E}$ corresponds to a $G$-invariant central idempotent $e$ of $\mathcal{E}$. This corresponds to an idempotent $\bar{e}$ of $\mathcal{E} / J_{G}(\mathcal{E}) \cong$ $\mathcal{E}[B] / J_{G[B]}(\mathcal{E}[B])$, which by Lemma 4.2 corresponds to an idempotent $e^{\prime}$ of $\mathcal{E}[B]$. As $J_{G[B]}(\mathcal{E}[B]) \subseteq J_{G}(\mathcal{E}), e$ and $e^{\prime}$ are central idempotents of $\mathcal{E}$ having the same image modulo $J_{G}(\mathcal{E})$, and so $e=e^{\prime}$.

The Clifford correspondence now follows immediately.

Theorem 4.5. Let $B$ be a $G$-invariant block of $A_{1}$. The blocks of $A$ covering $B$ correspond one-to-one with $G$-conjugacy classes of blocks of the twisted group algebra $\mathcal{E} / J_{G}(\mathcal{E})$. 
Proof. By Lemma 2.1 (ii) with $G=G_{B}$, Lemma 3.2, and Lemma 3.4, the blocks of $A$ covering $B$ correspond one-to-one with $G$-conjugacy classes of blocks of $\mathcal{E}$. By Lemma 4.4, $G$-conjugacy classes of blocks of $\mathcal{E}$ correspond one-to-one with $G$ conjugacy classes of blocks of $\mathcal{E} / J_{G}(\mathcal{E})$.

We close by returning to the general case where $B$ is a block of $A_{1}$ that is not necessarily $G$-invariant, and $G_{B}$ is the set of all elements $g$ of $G$ such that $A_{g^{-1}} B A_{g}=B$. By Lemma 2.2 and Theorem 4.5 with $G$ replaced by $G_{B}$, we have

Corollary 4.6. Let $B$ be a block of $A_{1}$ and $\mathcal{E}=\operatorname{End}_{A_{1}^{o p} \otimes A_{B}}\left(B \uparrow A_{1}^{o p} \otimes A_{B}\right)$. The blocks of $A$ covering $B$ correspond one-to-one with $G_{B}$-conjugacy classes of blocks of the twisted group algebra $\mathcal{E} / J_{G_{B}}(\mathcal{E})$.

\section{REFERENCES}

[1] J. L. Alperin and D. W. Burry, Block theory with modules, J. Algebra 65 (1980), pp. 225-233. MR 81k:20018

[2] D. J. Benson, Representations and Cohomology I: Basic representation theory of finite groups and associative algebras, Cambridge University Press, 1991. MR 92m:20005

[3] P. R. Boisen, The representation theory of fully group-graded algebras, J. Algebra 151 (1992), pp. 160-179. MR 93i:20005

[4] A. H. Clifford, Representations induced in an invariant subgroup, Ann. of Math. (2) 38 (1937), pp. 533-550.

[5] M. Cohen And S. Montgomery, Group-graded rings, smash products, and group actions, Trans. Amer. Math. Soc. 282 (1984), pp. 237-257. MR 85i:16002

[6] C. W. Curtis And I. Reiner, Methods of Representation Theory with Applications to Finite Groups and Orders, Volume I, Wiley, 1981. MR 82i:20001

[7] — Methods of Representation Theory with Applications to Finite Groups and Orders, Volume II, Wiley, 1987. MR 88f:20002

[8] E. C. DADE, Compounding Clifford's theory, Ann. of Math. 91 (1970), pp. 236-290. MR 41:6992

[9] — Block extensions, Illinois J. Math. 17 (1973), pp. 198-272. MR 48:6226

[10] — Group-graded rings and modules, Math. Z. 174 (1980), pp. 241-262. MR 82c:16028

[11] $—$ The equivalence of various generalizations of group rings and modules, Math. Z. 181 (1982), pp. 335-344. MR 84a:16018

[12] — Counting characters in blocks, I, Inv. Math. 109 (1992), pp. 187-210. MR 93g:20021

[13] _ Counting characters in blocks, II, J. reine angew. Math. 448 (1994), pp. 97-190. MR 95a:20007

[14] Clifford theory for blocks. Preprint, 1995.

[15] — Counting characters in blocks, 2.9, in Representation Theory of Finite Groups, Proceedings of a Special Research Quarter at the Ohio State University, de Gruyter, 1997. MR 99b:20016

[16] H. ElLers, Cliques of irreducible representations, quotient groups, and Brauer's theorems on blocks, Can. J. Math. 47 (5) (1995), pp. 929-945. MR 96g:20013

[17] W. FeIT, The Representation Theory of Finite Groups, North-Holland, 1982. MR 83g:20001

[18] J. Haefner, Graded equivalence theory with applications, J. Algebra 172 (1995), pp. 385424. MR 96f: 16052

[19] Y. Miyashita, On Galois extensions and crossed products, J. Fac. Sci. Hokkaido Univ. Ser. I 21 (1970), pp. 97-121. MR 42:6046

[20] C. Năstăsescu and F. van Oystaeyen, Graded Ring Theory, North-Holland, 1982. MR 84i: 16002

Department of Mathematics, University of Toronto, Toronto, Ontario, Canada M5S $3 \mathrm{G} 3$

E-mail address: sjw@math.toronto.edu

Current address: Department of Mathematics, University of Wisconsin, Madison, Wisconsin 53706

E-mail address: sjw@math.wisc.edu 